\title{
Modeling transcription factor binding events to DNA using a random walker/jumper representation on a 1D/2D lattice with different affinity sites
}

\author{
Vahid Rezania $^{1,2}$, Jack Tuszynski ${ }^{1}$ and Michael Hendzel ${ }^{1}$ \\ 1- Division of Experimental Oncology, Cross Cancer Institute \\ 11560 University Avenue, Edmonton, AB T6G 1Z2 Canada \\ 2 - Institute for Advanced Studies in Basic Sciences, Zanjan 45195, Iran. \\ E-mail: vrezania@phys.ualberta.ca
}

\begin{abstract}
Surviving in a diverse environment requires corresponding organism responses. At the cellular level, such adjustment relies on the transcription factors (TFs) which must rapidly find their target sequences amidst a vast amount of non-relevant sequences on DNA molecules. Whether these transcription factors locate their target sites through a $1 \mathrm{D}$ or 3D pathway is still a matter of speculation. It has been suggested that the optimum search time is when the protein equally shares its search time between $1 \mathrm{D}$ and $3 \mathrm{D}$ diffusions. In this paper, we study the above problem using a Monte Carlo simulation by considering a very simple physical model. A 1D strip, representing a DNA, with a number of low affinity sites, corresponding to non-target sites, and high affinity sites, corresponding to target sites, is considered and later extended to a $2 \mathrm{D}$ strip. We study the $1 \mathrm{D}$ and $3 \mathrm{D}$ exploration pathways, and combinations of the two modes by considering three different types of molecules: a walker that randomly walks along the strip with no dissociation; a jumper that represents dissociation and then re-association of a TF with the strip at later time at a distant site; and a hopper that is similar to the jumper but it dissociates and then re-associates at a faster rate than the jumper. We analyze the final probability distribution of molecules for each case and find that TFs can locate their targets fast enough even if they spend $15 \%$ of their search time diffusing freely in the solution. This indeed agrees with recent experimental results obtained by Elf et al. [1] and is in contrast with theoretical expectation.

Keywords: Transcription factor, DNA, Simulation, Search time
\end{abstract}




\section{Introduction}

The development of an adult animal from a single cell relies upon the ability of sequencetargeted DNA regulatory proteins to coordinate the expression of genes in a developmentand tissue-specific manner. These sequence-specific DNA binding transcription factors (TFs) must locate target sequences amidst a vast amount of non-relevant sequences. Surprisingly, the binding processes to specific sites happen at very fast rates [2], approximately 100-1000 times faster than the upper limit of a diffusion-controlled motion of molecules with the same size [3, 4. The mechanism(s) whereby these regulatory proteins find their target sites in long DNA molecules in such a rapid way has been the subject of extensive theoretical and experimental investigations. Yet it is currently still a matter of speculation. See review by Halford and Szczelkun [5].

In one scheme known as a 'sliding' or 'scanning' mechanism, the DNA binding protein binds randomly at any site on the DNA and then translocates along the sequence until it finds its target [6, 4]. In this scenario proteins move along the DNA by 1D diffusion-controlled motion without losing their contacts with the DNA. Each time the protein may move forward or backward (with equal probability) by taking one step along the DNA. This is equivalent to the symmetrical random walk problem in 1D.

The second mechanism, however, involves random walks between disassociation and reassociation events in solution. In this mechanism two different modes of behavior are available to proteins, namely the so-called 'hopping' and 'jumping' mechanisms, whereby proteins dissociate from the DNA and move through the solution by 3D diffusion-controlled motion before they can re-associate with another site on the same DNA [7, 8]. Hopping refers to the case when the re-association occurs at a distance, say, $\leq 20 \mathrm{bp}$ while jumping implies re-association at $>20 \mathrm{bp}$ from the dissociated site. Although hopping and jumping rates depends on the DNA topology (linear, folded, etc.), the overall rate of hopping would be higher than that of jumping 55. This is due to the low diffusivity of these macromolecules in solution (diffusion constant $\leq 10 \mu \mathrm{m}^{2} \mathrm{~s}^{-1}$ ), so that the majority of re-associations would happen at or very near to the dissociated site [6].

For those proteins with two or more binding sites (such as the Lac repressor, the SfiI and Ngo MIV endonucleases) on a single DNA molecule, 'intersegmental transfer' is considered as the third way that proteins move from one site to another. In this mechanism, a bound protein transiently binds to another site at the same time. After releasing, the protein may either remain at its initial binding site or move to a new site [9]. This mechanism, however, requires the juxtaposition of two sites within an interval shorter than the protein-DNA reaction radius in 3D space. It is shown that the intersegmental transfer mechanism is more likely for those proteins with two binding sites that are separate form each other by $>300$ bp [11, 10].

The sliding mechanism has been more attractive because of the assumption that diffusion along the length of the DNA is more rapid than a 3D search [2, 12, 6, 4, Several experimental strategies have been developed to determine whether or not the facilitated diffusion in 1D is the dominant mechanism. In one procedure, a group of DNA molecules of different lengths that each has a copy of the target-site is considered [14, 13, 16, 15, 4]. The results, however, did 
not rule out 3D diffusion (eg. hopping or jumping) and just showed that the protein binding association rate decreases as the DNA molecule gets shorter. This can be explained by noting that the binding protein has less chance to encounter a shorter DNA fragment [5].

In an alternative attempt, Terry et al [17] studied the binding of the EcoRI restriction enzyme on both linear and circular 388 bp DNA molecules (fixed length molecules) with two target-sites $51 \mathrm{bp}$ apart. Interestingly, the protein binding strength depends on whether the two target sites located closer to one end of DNA molecules or placed equidistant from both ends. In the former case, the enzyme showed more frequent cleavages at the innermost target site while in the latter case no preference between the target sites was observed. Binding to the circular DNA also showed no preference between the target sites, however, the degree of processivity was approximately two times higher than that on the linear DNA. Processivity is referred to as a fraction of total reactions that are cleaved at both target-sites during a single binding event. Strikingly, the processivity results show that translocation through the hopping (3D) mechanism must be more efficient than the sliding (1D) mechanism, otherwise the degree of processivity would be marginally above that on the linear DNA [8].

Combining the above strategies, Stanford and Szcselkun [8] measured the processivity factor for a group of DNA molecules with the same length but each consisted of two EcoRV target-sites that their inter-site spacing varied from one DNA to another ranging from 54 to $764 \mathrm{bp}$. Theoretically, by increasing the inter-site spacing, $n$, the processivity factor decreases with a rate of $n^{2}$ for $1 \mathrm{D}$ diffusion and $n^{1 / 2}$ for $3 \mathrm{D}$ diffusion [18. Interestingly, the resulting processivity factor did not match the 1D diffusion relationship (by several orders of magnitude) and more or less agreed with the 3D diffusion mechanism.

Halford and Szcselkun [5] also reviewed other experimental studies that were designed to determine whether a $1 \mathrm{D}$ or a 3D search is preferred by binding proteins and concluded that binding proteins find their target sites, both in vitro and in vivo, primarily through the 3D diffusion (hopping or jumping). This is, of course, in contrast to the perception that 1D diffusion along DNA molecules is the dominant pathway in locating target sites.

Several theoretical investigations, however, suggested that the optimum search time for a TF to locate its target site on the DNA strip is when the protein spends equal time sliding along the DNA and diffusing freely in the solution [7, 19]. A more precise calculation was carried out by Coppey et al. [20] who proposed a stochastic model for the protein-DNA interaction by considering a series of one dimensional diffusions of a single protein interrupted by several random jumps from site to site. They showed that for a DNA with an intermediate length the protein should spend a little bit more time (about $10 \%$ more) in the 1D pathway in order to minimize the search time. For a long DNA, however, they recovered the result by [7, 19].

More recently Elf et al. [1] performed an experimental investigation on lac repressor (LacI) in a living Escherichia coli cell and directly observed specific binding of the labeled lac repressor. Interestingly, using single-molecule tracking technique, they determined $1 \mathrm{D}$ and $3 \mathrm{D}$ diffusion constants of dimeric LacI-Venus to be $D_{1}=0.046 \pm 0.01 \mu \mathrm{m}^{2} \mathrm{~s}^{-1}$ (measured in vitro) and $D_{3}=3 \pm 0.3 \mu \mathrm{m}^{2} \mathrm{~s}^{-1}$ (measured in vivo and without its DNA biding domain), respectively. However, using the mean square displacement plot, they found that the effective diffusion constant of LacI in vitro is $D_{\text {eff }}=0.4 \pm 0.02 \mu \mathrm{m}^{2} \mathrm{~s}^{-1}$ that is 10 times bigger than 
1D constant. From this fact they concluded that TFs spend $87 \%$ of time sliding along DNA. This is obviously in contrast with theoretical results found in [7, 19, 20]. Furthermore, they measured the residence time of TFs on non-specific sites is about 0.3 to $5 \mathrm{~ms}$ and the searching time for a single TF to find a target site is about 65 to $360 \mathrm{sec}$. The residence time corresponds with probability of staying TF in a particular site.

The above result is more or less agrees with a single molecule spectroscopy done by Pant et al. [21, 22] and Sokolov et al. [23]. They studied the relation of the protein binding rate with the protein concentration, $C$, in the solution. They argued that the rate of binding to the specific site is proportional to $C^{2}$ and $C$ for pure 1D and 3D diffusion, respectively. By considering bacteriophage T4 gene 32 protein, Pant et al. 21, 22] found that at low to moderate protein concentration the binding rate to the specific site of a single-stranded DNA is more or less proportional to the $C^{2}$. This shows that most of the time the protein bounded to the DNA.

In this study we perform a numerical simulation of the interaction of binding proteins with DNA molecules. Our aim is to elucidate through numerical modeling whether a 1D or a 3D pathway is more favorable and type of results can be expected from a combination mode of behavior. Here the 1D pathway refers to the case that molecules move along the lattice without disassociation from the lattice while the 3D pathway refers to the case that molecules disassociate and then re-associate as they move from site to site. We consider a binding protein as a random walker (hopper/jumper) that walks (hops/jumps) along a one or two-dimensional lattice representing DNA molecules. Binding to a site depends on the binding energy between the molecule and the site that is represented here as an affinity to the site. The smaller the affinity the bound molecule has the greater the chance to translocate to another site. In general, each site may have a different affinity from others. In this study, however, we assume two different affinities for simplicity: low affinity (LA) sites and high affinity (HA) sites. The generalization to several non-equal affinity sites is straightforward. The HA sites can be considered as target (specific) sites that a binding protein is searching for. The LA sites are those non-specific sites that a protein moves along them to find its target site. Furthermore, we assume that each site can have up to a single molecule bound to it at a given time.

It is necessary to note that based on several experimental studies the model considered here, though is very simplistic, is a good approximation for prokaryote (and possibly eukaryote) cells [1, 21, 22, 23].

\section{Method}

To represent the DNA substrate for TF binding, consider a one dimensional lattice made of $L$ sites (in total) comprised of low affinity (LA) and high affinity (HA) sites. Such a system can be achieved, for example, by pulling DNA with optical tweezers to avoid DNA folding [21, 22, 23]. A molecule, representing a TF, may leave an LA site with a greater probability than an HA site. As a result, the probability of finding a molecule in an HA site is greater than that in an LA site. In terms of the binding energies, these sites can be represented by several potential wells placed in sequence along the DNA lattice in which HA sites have deeper potentials [19].

See Fig. 1. Here, we assume that $10 \%$ of the of total number of accessible sites to TF proteins 
are HA sites distributed among LA sites in an a priori unknown fashion which is reasonable assumption that can be verified by inspecting actual DNA sequence data.

The probability of leaving from an LA or HA site can be estimated by observing the residence time of a binding protein to non-specific or specific sites. Using the fluorescent recovery after photobleaching (FRAP) technique, the mean residence times for several chromatin-binding proteins have been studied [24, 25, 26]. The reader is referred to the review by van Holde and Zlatanova [27] for further references. Phair et al. [26] studied over 20 chromatin proteins and distinguished two slow and fast recovery populations with mean residence times ranging from $\sim 3$ to $6 \mathrm{sec}$ for the fast population and from $\sim 15$ to $30 \mathrm{sec}$ for the slow population. They found Jun and XBP proteins have the shortest mean residence time of $\sim 2$ sec and $\mathrm{H} 1^{0}$ has the longest one $\sim 3$ min. See Tables 1-3 in the paper by Phair et al. [26]. Elf et al. [1] also measured the residence time in non-specific sites is about $5 \mathrm{~ms}$. Here, we assumed that the molecule has a $20 \%$ chance to leave an HA site while it has a $67 \%$ chance to leave LA sites. We assume that the probability of leaving a particular binding site, $P$, is related to the binding energy, $E$ via an Arrhenius relationship: $P=P_{0} \exp (-\Delta E / k T)$. Here $k$ is the Boltzmann's constant and $T$ is the temperature. Hence the choice of the probability values for LA and HA sites corresponds to an energy difference between then of approximately $1.2 k T$ which is at the low end of the expected levels. Energy differences of less than $k T$ would not results in a high- and low-affinity designation.

As we mentioned earlier, a molecule can walk or hop/jump along the lattice. A walker moves one step at a time without dissociating from the lattice. The walker moves left or right with an equal probability (symmetric walker). In the case of jumpping, however, one molecule dissociates from a site (LA or HA) and after a random time interval, the same or another molecule associates at any available site (chosen randomly) along the lattice. The hopper, in contrast with jumper, re-associates to the lattice instantly (if the free site is available) within a 100-site radius from the dissociated site. As a result, the hopper does not travel too far from the dissociated site. For the hopper/jumper there is no left or right preference. In the Appendix we have given background information and mathematical formulas for the probability distributions for random walkers and random jumpers. The reason for our choice of a random reassociation site is due to the typical range of values for the $3 \mathrm{D}$ diffusion constant which is on the order of $1 \mu \mathrm{m}^{2} / \mathrm{s}$. Together with the formula: $\left\langle x^{2}\right\rangle=6 D t$ and taking the value of $t$ on the order of $1 \mathrm{~s}$ gives the rms distance traveled in the surrounding medium on the order of $2-3 \mu \mathrm{m}$ which indicates that the freed TF can find itself in an arbitrary location along the DNA upon rebinding.

The simulation starts from an initial state made of $M(<L)$ molecules that are randomly distributed along the lattice. For the walking case, a random number $r$ is chosen and then compared with the site's probability, $P_{0}=\left(P_{\mathrm{HA}}\right.$ or $\left.P_{\mathrm{LA}}\right)$. The site's probability is plotted schematically in Fig. 1. As seen, $100 \mathrm{HA}$ sites are clustered in the middle of the lattice with a total $L=1000$ sites. If $r>P_{0}$ and its immediate neighbor is free, the walker will leave, otherwise it will stay. The immediate left or right neighbor is also chosen randomly using a separate uniform random distribution. Note that here we do not assume that the molecule is trapped in the HA for good. This assumption can change the final result drastically. We 
will back to this point later. Similarly, in the case of hopping/jumping, a random number $r$ is chosen and then compared with the site's probability, $P_{0}=\left(P_{\mathrm{HA}}\right.$ or $\left.P_{\mathrm{LA}}\right)$. In this case only, a pool of molecules is also considered such that a new molecule from the pool may associate to a free site on the lattice and a molecule may dissociate from the lattice and enter the pool. The jumper will leave the site when $r>P_{0}$ and enter the pool of free TF's, otherwise it will stay bound to the DNA. After a random time interval, a destination site is chosen randomly using a separate uniform random distribution. If the new site is free, a molecule from the pool will associate to the DNA. In the case of hopping, the new destination site is chosen within a 100 -site radius from the disassociated site with a diminishing probability with a distance. As a result, the adjacent sites to the the disassociated site are more probable. The above procedure is repeated $N_{\text {sim }}$ times.

To represent the problem more realistically, we also study the dynamics of TF's on a 2D lattice consisting of several affinity sites. In this case, in addition to LA and HA sites, a zero affinity site representing solution (water) is also considered. See Fig. 2, As seen in Fig. 2, as well as individual HA sites, there are some clustered HA sites along $\mathrm{x}$ - and $\mathrm{y}$-direction. Here $\mathrm{x}$-direction refers to the motion along a particular DNA molecule and $\mathrm{y}$-direction refers to the motion along different DNA molecules. The clustered HA sites in y-direction represent individual HA sites from different DNA molecules. This type of clustering, in fact, have been observed experimentally and it supports our model.

\section{Results}

In total, over the course of our simulation each molecule, a walker or a jumper, will have $N_{\text {step }}$ (successful or unsuccessful) movement events. Note that each step represents a unit of time in the simulation. In general we are interested in the total time (or step) that takes the system reaches to the equilibrium. It is clear that the equilibrium distribution occurs when all the HA sites are filled. To have a better statistic, the simulation runs for $N_{\text {sim }}$ times. Here, the final state for walkers (jumpers) is found after $N_{\text {step }}=1000,10000,50000$ and 100000 steps (10, 100 and 1000), each with $N_{\text {sim }}=1000$. The results are shown in Figs. 3, 8 .

In Figs. 3 and 4 we assume that the $100 \mathrm{HA}$ sites are clustered in the middle. Figure 3 demonstrates the final distribution of the 105 walkers after $N_{\text {step }}=1000$ steps (blue circle), 10000 steps (green diamond), 50000 steps (red asterisk) and 100000 steps (black star). The distribution is fairly uniform everywhere except close to the HA sites. The distribution decreases near the edges, suddenly increases at the edges and then decreases as it goes toward the middle. Interestingly, not all the HA sites have the highest probability, except those who placed at the edges. Figure 4 presents the probability of finding a randomly distributed ensemble of 105 hopper/jumpers after $N_{\text {step }}=10$ steps (blue circle), 100 steps (red asterisk), 1000 steps (green diamond) in the lattice. It is clear that the equilibrium distribution is obtained after $N_{\text {jump }} \sim 100$ jumps. As expected, all the HA sites have the highest probability which is interestingly constant (more or less) for all curves. The LA sites, however, are shown to have more or less similar probabilities for each run with a limiting value $\sim 0.33=1-P_{0}$. Interestingly, the walker shows very different behaviors at the final 
state in comparison with jumpers. It is clear that the longer the molecules walk or jump, the closer the final distribution approximates an equilibrium distribution where it should be $P_{N_{\text {step }}}(x) \simeq P_{\text {equilibrium }}(x)=1-P_{0}(x)$ [28]. Comparing Figs. 3 and 4 one can say the hoppers/jumpers reach the final distribution in a much shorter time (equivalently smaller steps) than walkers. As seen, even after $N_{\text {walk }}=100,000$ steps, the final distribution of walkers does not match the equilibrium distribution completely. More interestingly, the square root relation is also observed between the required number of jump events and number of walk events in order to reach the equilibrium distribution, i.e. $N_{\text {jump }} \sim \sqrt{N_{\text {walk }}}$. This is in agreement with observations [8].

The difference between the 1D (walking) and 3D (jumping) pathways is also studied for different LA and HA sites distributions. Figure 5 demonstrates final distributions of walkers and jumpers when 50 single HA sites are distributed among LA sites. In this case the none of the HA sites is clustered. Figure 6 represents the results for randomly distributed sites with different depths. Though in all cases the jumpers reach the equilibrium distribution in a shorter time scale, for single HA site distribution (Fig. 5) the walkers reach the equilibrium distribution faster in comparison with the cases when the HA sites are clustered.

The above results can be explained by noting that the pure jumping case is equivalent with a system with high protein concentration. So there are enough TFs around the DNA to bind the target sites immediately. In contrast, in the case of pure walking, the protein concentration of the solution is zero. So TFs will find their target sites after scanning more or less all the lattice. To be more realistic, we consider a mixed case. Starting from the case of (100\% walker, $0 \%$ jumper), we increase the jumper's percentage in the system. Interestingly, we find that after having a mixture of ( $85 \%$ walker, $15 \%$ jumper), the equilibrium distribution can be achieved after $N_{\text {step }} \sim 100-1000$ steps. This agrees with the experimental results found by Elf et al. [1] and contrasts with the theoretical expectation [7, 19, 20]. In Fig. 7 we demonstrate the result of three different mixtures of random walkers and jumpers, $(95 \%, 5 \%),(90 \%, 10 \%)$ and $(85 \%$, $15 \%$ ) after $N_{\text {step }}=100$. As a result, if TFs spend about $15 \%$ of their time diffusing freely in the solution, they can find their target sites in a very short time.

In the above simulations we assumed that the molecules can escape from the HA sites (though, with smaller probability) and so they would not be trapped in the HA sites for good after entering those sites. In order to see how this assumption would affect the results, we run some simulations by assuming molecules will be trapped for good after entering HA sites. Interestingly, we find that the equilibrium distribution cannot be achieved in a short time (100-1000 steps) unless more than $80 \%$ of the molecules are jumpers.

It is generally known that a symmetric random walk distribution approaches the Gaussian distribution after a long run, i.e $N_{\text {step }} \rightarrow \infty$. This is a consequence of the the central limit theorem. In general, the required number of steps to achieve a desire level of convergence to the equilibrium distribution can be estimated using the Berry-Esséen theorem [29] as

$$
N \geq \frac{25}{4} \frac{\left\langle|x|^{3}\right\rangle^{2}}{\left\langle x^{2}\right\rangle^{3}} \frac{1}{\epsilon^{2}},
$$

where $\epsilon$ represents the level of convergence. It is clear that the criterion 1 relies on the ratio between the second, $\left\langle x^{2}\right\rangle$, and third, $\left\langle|x|^{3}\right\rangle$, moments of the random walk/jump probability 
distribution. In the Appendix we calculate the ratio $\left\langle|x|^{3}\right\rangle /\left\langle x^{2}\right\rangle^{3 / 2}$ for three different cases: a random walk probability $p_{\mathrm{w}}(x)$, a random jump probability $p_{\mathrm{j}}(x)$, and a mixed probability $p_{\mathrm{wj}}(x)$, with results of $1.000,1.2990$ and 1.1482 , respectively. Figure 8 represents $\left\langle|x|^{3}\right\rangle /\left\langle x^{2}\right\rangle^{3 / 2}$ ratio as function of $N_{\text {step }}$ for 105 walkers, jumpers, hopper and/or mixed for the initial clustered distribution (see Fig. 1). The results for all runs are in the range of $1.3 \pm .03$ which is close to the random jump distribution .

As mentioned earlier, in order to make the model more realistic, we also performed several simulations with a 2D lattice (Fig. 22). In general, one would expect several DNA molecules are present in the solution at different locations. As a result, TF's that are moving in the bulk have now more chances to bind. Similarly to 1D lattice, we consider two different molecules: walker and hopper/jumper. We assume that a walker can only walk along the DNA (x-direction) except when it encounters zero affinity sites. In those sites, the walker can move in both $\mathrm{x}$ and y- directions. Hoppers/jumpers can move in both directions with no restriction. We used similar strategy for walking and hopping/jumping from one site to another as we employed in the 1D lattice (see above). Results are shown in Figs. 9r11.

Figure 9 shows the probability distribution of 120 walkers after walking for 100, 10, 000 and 100,000 steps. To compare, we also show the expected final distribution of molecules $P_{\text {equilibrium }}(x, y) \sim 1-P_{0}(x, y)$ for given probability in Fig. 2, The probability distribution of 120 jumpers after jumping for 100 steps is shown in Fig. 10. Comparing with the expected distribution, it is clear that the jumpers reach to the equilibrium distribution in a shorter time (smaller steps) than walkers.

Figure 11 demonstrates the probability distribution of 120 mixed walkers and jumpers after walking/jumping for 1000 steps. As seen, by increasing the number of jumpers, the final distribution approaches to the equilibrium distribution. This is again in good agreement with the experimental results obtained by Elf et al. [1].

\section{Discussion}

It is well known that very complicated processes such as the coordination of gene expression, cellular metabolism, and organ and tissue development rely on a more fundamental process, the regulation of gene transcription through transcription factor binding. This process coordinates the expression of genes in a tissue-specific manner during early stages of development and tissue specification and is crucial in the development of an adult animal from a single cell. The fundamental question is how do these transcription factors find their target sites amongst a large number of non-target sites. This is still a matter of speculation. Specifically, experimental results suggest that transcription factors must locate target sequences at very fast rates [2], approximately 100-1000 times faster than the upper limit of a diffusion-controlled motion of molecules with the same dimension [3, 4. An enormous amount of effort has been spent trying to understand whether transcription factors locate their targets through a 1D (sliding along the DNA ) or 3D (diffusion in solution) pathway. Though earlier investigations favored the $1 \mathrm{D}$ pathway, recent experimental studies suggest that the mixture of $1 \mathrm{D}$ and 3D pathways is more efficient [1]. 
In this paper we address the above question using a Monte Carlo simulation. We consider a 1D strip with a number of potential wells at different depths that are clustered on the strip. The strip represents a DNA molecule and each potential well represents a binding site. In the simplest case we distinguish two potential depths: low affinity (LA) sites with shallow potential and high affinity (HA) sites with deep potential. See Fig. 1. The generalization to several potential depths is straightforward.

The strip is located in a pool of molecules (a 2D lattice with zero affinity sites) that can associate with or dissociate from it. In our simulations, however, we consider three different molecules: a walker that performs a random walk along the strip without dissociation from it; a jumper that dissociates from the strip and then re-associates with the strip at a distant site after a random time interval; and a hopper that dissociates and then re-associates at a faster rate than the jumper. As a result, the hopper travels less distance than the jumper before re-association. The walker represents a binding protein that slides along the DNA to find its target site via the 1D pathway. The jumper, in contrast, is a protein that uses the 3D pathway (free diffusion in the solution) to locate its target site on the DNA.

Several simulations with different molecules and different number of steps are performed: walkers only with $N_{\text {step }}=1000,10,000,50,000$ and 100,000; jumpers/hoppers only with $N_{\text {step }}=10,100$ and 1000; and a mixture of walkers, jumpers and hoppers. Our results are plotted Figs. 3 3, 8 , As seen in Figs. 3 and 4, jumpers reach the equilibrium distribution after order of $N_{\text {jump }} \sim 100$ steps while the final distribution of walkers even after $N_{\text {walk }}=100,000$ steps is quite different from the expected equilibrium distribution. Interestingly, we find that $N_{\text {jump }} \sim \sqrt{N_{\text {walk }}}$, which is in good agreement with observations [8]. In the case of mixed molecules, we examined different populations of molecules and found that even if $15 \%$ of the molecule population are not walkers, the results are very similar to the jumper case. See Fig. 7 that is shown the result of three different mixtures of random walkers and jumpers, $(95 \%, 5 \%)$, $(90 \%, 10 \%)$ and $(85 \%, 15 \%)$ after $N_{\text {step }}=100$. Our results show that if the TFs spend just about $15 \%$ of their search time diffusing in the solution they are able to locate their target site in a short period of time. This indeed agrees with experimental results obtained in [21, 22, 23. and specifically by Elf et al. [1] who found $87 \%$ of the time TFs are diffusing along the DNA. However, our results are in contrast with the theoretical expectation that suggests the optimum search time happens when the protein spends equal time sliding along the DNA and diffusing freely in the solution (a 50-50 mixture) [7, 19, 20].

As expected, similar results are also obtained when we consider a $2 \mathrm{D}$ lattice rather than a simple 1D lattice (Fig. 2). It is more realistic to assume several DNA molecules are present in the solution at different locations. As a result, TF's that are moving in the bulk have now more chances to bind. Results are shown in Figs. 9, 11. We compared the probability distribution of 120 walkers after walking for 100, 10,000 and 100,000 steps with that of 120 jumpers after jumping for 100 steps as shown in Figs. 9 and 10. It is clear that the jumpers reach to the equilibrium distribution in a shorter time (smaller steps) than walkers.

In recent years, however, some experimental investigations suggest a new role for chromatin in transcription factor regulation. Chromatin consists of a fundamental unit called the nucleosome, a disc-shaped octamer of eight histone proteins, bound to DNA. Each histone 
interacts with other histones and DNA to form the nucleosome. A chromatin-medited mechanism may enhance fidelity of transcriptional regulation and control of gene expression in the cell. Initial experiments monitoring histone H1-GFP fusion during FRAP experiments have shown a rapid exchange of the $\mathrm{H} 1$ with sites (with few minutes residence time) while being statically fixed on chromatin 24, 25. A number chromatin-binding proteins have subsequently been monitored by Phair et al. 26] in vivo using FRAP method. They found that transient binding is a common property among chromatin-associated proteins. They also demonstrated that all of these chromatin-binding proteins show rapid mobility. They concluded that these proteins continuously scan the genome space for appropriate binding sites through diffusional hopping (the 3D pathway) between chromatin fibers. Buck and Leib [31] also studied repressoractivator protein 1 in the yeast cells and found that these proteins locate their binding sites via a newly dynamic target specification mechanism. Chromatin-mediated regulation of accessibility coordinates genome-wide distribution of DNA sequence motifs to target sites by remodeling the genome itself.

\section{Acknowledgments}

This research was supported by grants from the ACB, NSERC, CSA and CHIR. Support from the Alberta Foundation is gratefully acknowledged by J. A. T. We thank Romain Sibi for his assistance with some of our numerical simulations.

\section{Appendix A. Calculating $\left\langle|x|^{3}\right\rangle /\left\langle x^{2}\right\rangle^{3 / 2}$ for different distribution functions}

\section{Appendix A.1. Random walk distribution}

The distribution function for a non-equal step size random walk in the range $[-\ell, \ell]$ can be written as

$$
p_{\mathrm{w}}(x)=a \delta(x+\ell)+b \delta(x-\ell),
$$

where $\delta(x)$ is the Dirac delta function and $a+b=1$. The $n^{\text {th }}$ moment can be calculated as

$$
\begin{aligned}
\left\langle x^{n}\right\rangle & =\int_{-\infty}^{\infty} x^{n} p_{\mathrm{w}}(x) d x, \\
& =\int_{-\infty}^{\infty} x^{n}(a \delta(x+\ell)+b \delta(x-\ell)) d x, \\
& =\left[(-1)^{n} a+b\right] \ell^{n},
\end{aligned}
$$

and

$$
\begin{aligned}
\left\langle|x|^{n}\right\rangle & =\int_{-\infty}^{\infty}|x|^{n} p_{\mathrm{w}}(x) d x, \\
& =\int_{-\infty}^{\infty}|x|^{n}(a \delta(x+\ell)+b \delta(x-\ell)) d x, \\
& =a \ell^{n}+b \ell^{n}=\ell^{n},
\end{aligned}
$$

Therefore, for the random walk distribution we have

$$
\begin{aligned}
& \left\langle|x|^{3}\right\rangle=\ell^{3}, \\
& \left\langle x^{2}\right\rangle=\ell^{2} .
\end{aligned}
$$


As a result,

$$
\frac{\left\langle|x|^{3}\right\rangle}{\left\langle x^{2}\right\rangle^{3 / 2}}=1
$$

\section{Appendix A.2. Random jump distribution}

The distribution function for a random jump in the range $[-L / 2, L / 2]$ can be written as

$$
p_{\mathrm{j}}(x)=(1 / L)(H(x+L / 2)-H(x-L / 2)),
$$

where $H(x)$ is the Heaviside function:

$$
H(x)= \begin{cases}0 & x<0 \\ 1 & x \geq 0\end{cases}
$$

The $n^{\text {th }}$ moment can be calculated as

$$
\begin{aligned}
\left\langle x^{n}\right\rangle & =\int_{-\infty}^{\infty} x^{n} p_{\mathrm{j}}(x) d x \\
& =(1 / L) \int_{-\infty}^{\infty} x^{n}(H(x+L / 2)-H(x-L / 2)) d x \\
& =\frac{\left[1+(-1)^{n}\right](L / 2)^{n}}{2(n+1)},
\end{aligned}
$$

and

$$
\begin{aligned}
\left\langle|x|^{n}\right\rangle & =\int_{-\infty}^{\infty}|x|^{n} p_{\mathrm{j}}(x) d x \\
& =(1 / L) \int_{-\infty}^{\infty}|x|^{n}(H(x+L / 2)-H(x-L / 2)) d x \\
& =\frac{(L / 2)^{n}}{(n+1)} .
\end{aligned}
$$

Therefore, for the random jump distribution we have

$$
\begin{aligned}
& \left\langle|x|^{3}\right\rangle=(1 / 4)(L / 2)^{3}, \\
& \left\langle x^{2}\right\rangle=(1 / 3)(L / 2)^{2} .
\end{aligned}
$$

As a result,

$$
\frac{\left\langle|x|^{3}\right\rangle}{\left\langle x^{2}\right\rangle^{3 / 2}}=\frac{3^{3 / 2}}{4}=1.2990
$$

\section{Appendix A.3. Random walk-jump distribution}

The distribution function for a mixed random walk-jump in the range $[-L / 2, L / 2]$ where $\ell \leq L / 2$ can be written as

$$
\begin{aligned}
p_{\mathrm{wj}}(x) & =\alpha p_{\mathrm{j}}(x)+\beta p_{\mathrm{w}}(x), \\
& =\frac{\alpha}{L}(H(x+L / 2)-H(x-L / 2))+\beta(a \delta(x+\ell)+b \delta(x-\ell)),
\end{aligned}
$$


where $\alpha+\beta=1$. The $n^{\text {th }}$ moment can be calculated as

$$
\begin{aligned}
\left\langle x^{n}\right\rangle & =\int_{-\infty}^{\infty} x^{n} p_{\mathrm{wj}}(x) d x, \\
& =\frac{\alpha\left[1+(-1)^{n}\right](L / 2)^{n}}{2(n+1)}+\beta\left[(-1)^{n} a+b\right] \ell^{n},
\end{aligned}
$$

and

$$
\begin{aligned}
\left\langle|x|^{n}\right\rangle & =\int_{-\infty}^{\infty}|x|^{n} p_{\mathrm{wj}}(x) d x, \\
& =\frac{\alpha(L / 2)^{n}}{(n+1)}+\beta \ell^{n} .
\end{aligned}
$$

Therefore, for the random walk-jump distribution we have

$$
\begin{aligned}
& \left\langle|x|^{3}\right\rangle=(\alpha / 4)(L / 2)^{3}+\beta \ell^{3}, \\
& \left\langle x^{2}\right\rangle=(\alpha / 3)(L / 2)^{2}+\beta \ell^{2} .
\end{aligned}
$$

As a result,

$$
\frac{\left\langle|x|^{3}\right\rangle}{\left\langle x^{2}\right\rangle^{3 / 2}}=\frac{(\alpha / 4)(L / 2)^{3}+\beta \ell^{3}}{\left[(\alpha / 3)(L / 2)^{2}+\beta \ell^{2}\right]^{3 / 2}} .
$$

For a special case $\alpha=1 / 2=\beta$ and $\ell=L / 2$, we find $\left\langle|x|^{3}\right\rangle /\left\langle x^{2}\right\rangle^{3 / 2}=1.1482$.

\section{References}

[1] Elf J, Li G, and Xie XS 2007 Probing transcription factor dynamics at the single-moleule level in a living cell. Science 316 1191-1194.

[2] Riggs AD, Burgeoise S, and Cohn M 1970 The lac repressor-operator interaction. 3. Kinetic studies. J. Mol. Biol. 53 401-17.

[3] Berg OG, and von Hippel PH 1985 Diffusion-controlled macromolecular interactions. Annu. Rev. Biophys. Chem. 14 131-60.

[4] Shimamoto N 1999 One-dimensional diffusion of proteins along DNA. Its biological and chemical significance revealed by single-molecule measurements. J. Biol. Chem. 274 15293-6.

[5] Halford E, and Szczelkun MD 2002 How to get from A to B: strategies for analysing protein motion on DNA. Eur. Biophys. J. 31 257-6.

[6] von Hippel PH, and Berg OG 1989. Facilitated target location in biological systems. J. Biol. Chem. 264 675-8.

[7] Berg OG, Winter RB, and von Hippel PH 1981 Diffusion-driven mechanisms of protein translocation on nucleic acids. 1. Models and theory. Biochemistry 20 6929-48.

[8] Stanford NP, Szczelkun MD, Marko JF, and Halford SE 2000 One- and three-dimensional pathways for proteins to reach specific DNA sites. EMBO J. 19 6546-57.

[9] Milsom SE, Halford SE, Embleton ML, and Szczelkun MD 2001 Analysis of DNA looping interactions by type II restriction enzymes that require two copies of their recognition sites. J. Mol. Biol. 311 515-27.

[10] Podtelezhnikov AA, and Vologodskii AV 2000 Dynamics of small loops in DNA molecules. Macromolecules 33 2767-2771.

[11] Ringrose L, Chabanis S, Angrand PO, Woodroofe C, and Stewart AF 1999 Quantitative comparison of DNA looping in vitro and in vivo: chromatin increases effective DNA flexibility at short distances. EMBO J. 18 6630-41.

[12] Richter PH, and Eigen M 1974 Diffusion controlled reaction rates in spheroidal geometry. Application to repressor-operator association and membrane bound enzymes. Biophys Chem. 2 255-63. 
[13] Ehbrecht HJ, Pingoud A, et al. 1985 Linear diffusion of restriction endonucleases on DNA. J. Biol. Chem. $2606160-6$

[14] Jack WE, Terry BJ, and Modrich P 1982 Involvement of outside DNA sequences in the major kinetic path by which EcoRI endonuclease locates and leaves its recognition sequence. Proc. Natl. Acad. Sci. US A. 79 4010-4.

[15] Jeltsch A, and Pingoud A 1998 Kinetic characterization of linear diffusion of the restriction endonuclease EcoRV on DNA. Biochemistry. 37 2160-9.

[16] Jeltsch A, Wenz C, Stahl J, and Pingoud A 1996 Linear diffusion of the restriction endonuclease EcoRV on DNA is essential for the in vivo function of the enzyme. EMBO J. 15 5104-11.

[17] Terry BJ, Jack WE, and Modrich P 1985 Facilitated diffusion during catalysis by EcoRI endonuclease. Nonspecific interactions in EcoRI catalysis. J. Biol. Chem. 260 13130-7.

[18] Doi M, and Edwards SF 1984 The theory of polymer dynamics. Oxford University Press, Oxford.

[19] Slutsky M, and Minry LA 2004 Kinetics of protein-DNA interaction: facilitated target location in sequencedependent potential. Biophys. J. 87 4021-4035.

[20] Coppey M, Benichou O, Voituriez R, and Moreau M 2004 Kinetics of target site localization of a protein on DNA: a stochastic approach. Biophys. J. 87 1640-1649.

[21] Pant, K., R. L. Karpel, L. Rouzina, and M. C. Williams. 2004. Mechanical Measurement of Single-molecule Binding Rates: Kinetics of DNA Helix-destabilization by T4 Gene 32 Protein. J. Mol. Biol. 336:851870.

[22] Pant, K., R. L. Karpel, L. Rouzina, and M. C. Williams. 2005. Salt Dependent Binding of T4 Gene 32 Protein to Single and Double-stranded DNA: Single Molecule Force Spectroscopy Measurements. J. Mol. Biol. 349:317330.

[23] Sokolov IM, Metzler R, Pant K, and Williams MC 2005 Target Search of N sliding proteins in a DNA. Biophys. J. 89 895-902.

[24] Lever MA, Th'ng JPH, Sun X, and Hendzel MJ 2000. Rapid exchange of histone H1.1 on chromatin in living human cells. Nature. 408 873-6.

[25] Misteli T, Gunjan A, Hock R, Bustin M, and Brown DT 2000 Dynamic binding of histone H1.1 to chromatin in living cells. Nature. 408877.

[26] Phair RD, Scaffidi P, Elbi C, et al. 2004 Global nature of dynamic protein-chromatin interactions in vivo: three-dimansional genome scanning and dynamic interaction networks of chromatin proteins. Mol. Cell. Biol. 246393.

[27] van Holde K, and Zlatanova J 2006 Scanning chromatin: a new paradigm? J. Biol. Chem. 28112197.

[28] van Kampen NG 1981 Stochastic Processes in Physics and Chemistry. North-Holland Publishing Co., Amsterdam, Chap. VIII, p. 209.

[29] Feller W 196. An Introduction to Probability Theory and Its Application. John Wiley \& Sons Inc., New York, Vol. 2, Cahp. XVI.8, p. 525.

[30] Santos-Rosa H, Schneider R, Bannister AJ, et al. 2002 Active genes are tri- methylated at K4 of histone H3. Nature. 419 407-11.

[31] Buck MJ, and Lieb JD 2006 A chromatin-mediated mechanism for specification of conditional transcription factor targets. Nat Genet. 38 1446-51.

\section{Figure Legends}

Figure 1. The leaving probability distribution of $L=1000$ sites. $100 \mathrm{HA}$ sites are clustered in the middle.

Figure 2. The leaving probability distribution $P_{0}(x, y)$ of 2000 sites in a $2 \mathrm{D}$ lattice. About $70 \%$ of sites are red sites representing the solution that have zero affinity. The x-direction specifies the direction along a particular DNA molecule and the y-direction shows the direction along different DNA molecules. 
Figure 3. Probability distribution of 105 walkers after walking for 1000 steps (circle), 10, 000 steps (diamond), 50,000 steps (asterisk) and 100,000 steps (star). The HA sites are clustered in the middle.

Figure 4. Probability distribution of 105 jumpers after jumping for 10 steps (circle), 100 steps (asterisk), and 1000 steps (diamond). The HA sites are clustered in the middle.

Figure 5. (a) Initial distribution of the 50 single HA sites (not clustered) with different depths. (b) Probability distribution of 105 jumpers after jumping for 100 steps for the initial distribution in (a). Probability distribution of 105 walkers after walking for (c) 100 steps and (d) 10000 steps for the distribution in (a).

Figure 6. (a) Initial randomly distributed binding sites with non-equal depth. (b) Probability distribution of 105 jumpers after jumping for 100 steps for the initial distribution in (a). (c) Initial randomly distributed binding sites with non-equal depth. (d) Probability distribution of 105 walkers after walking for 10000 steps for the initial distribution in (c).

Figure 7. Probability distribution of 105 mixed walkers and jumpers after 100 steps. Circle: (95\% walker, 5\% jumper), diamond: (90\% walker, 10\% jumper), and asterisk: (85\% walker, $15 \%$ jumper). The HA sites are clustered in the middle.

Figure 8. $\quad\left\langle|x|^{3}\right\rangle /\left\langle x^{2}\right\rangle^{3 / 2}$ value as function of number of steps for 105 walkers, jumpers, hoppers and/or mixed molecules. The $100 \mathrm{HA}$ sites are clustered in the middle. Blue line-circle: walker with $N_{\text {step }}=5000$; red line-circle: walker with $N_{\text {step }}=10,000$; black line-circle: walker with $N_{\text {step }}=50,000$; green line-circle: walker with $N_{\text {step }}=100,000$; blue line-square: jumper with $N_{\text {step }}=5,000$; red line-square: jumper with $N_{\text {step }}=10,000$; blue line-triangle: hopper with $N_{\text {step }}=5000$; black line-triangle: mixed ( $50 \%$ walker, $25 \%$ jumper, $25 \%$ hopper) with $N_{\text {step }}=5000$; purple line-triangle: mixed (80\% walker, $10 \%$ jumper, $10 \%$ hopper) with $N_{\text {step }}=5000$. Those data with $N_{\text {step }}>5,000$ are scaled.

Figure 9. (a) Expected final distribution of molecules for given probability in Fig. 2. Probability distribution of 120 walkers after walking for (b) 100 steps, (c) 10, 000 steps and (d) 100, 000 steps.

Figure 10. (a) Expected final distribution of molecules for given probability in Fig. 2, (b) Probability distribution of 120 jumpers after jumping for 100 steps.

Figure 11. (a) Expected final distribution of molecules for given probability in Fig. 2. Probability distribution of 120 mixed walkers and jumpers after walking/jumping for 1000 steps. (b) $80 \%$ walkers with $20 \%$ jumpers. (c) $50 \%$ walkers with $50 \%$ jumpers. (d) $20 \%$ walkers with $80 \%$ jumpers. 


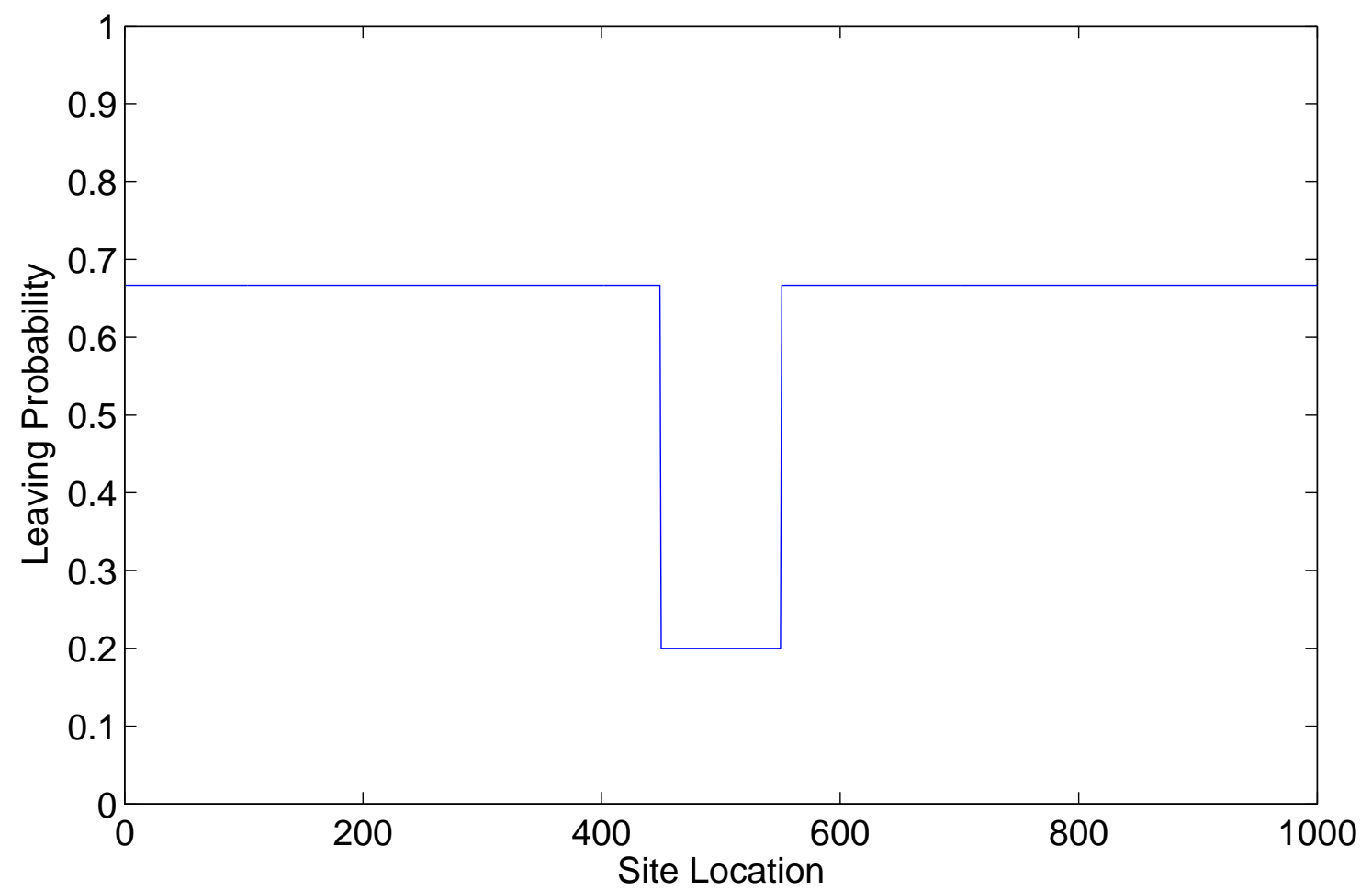

Figure 1.

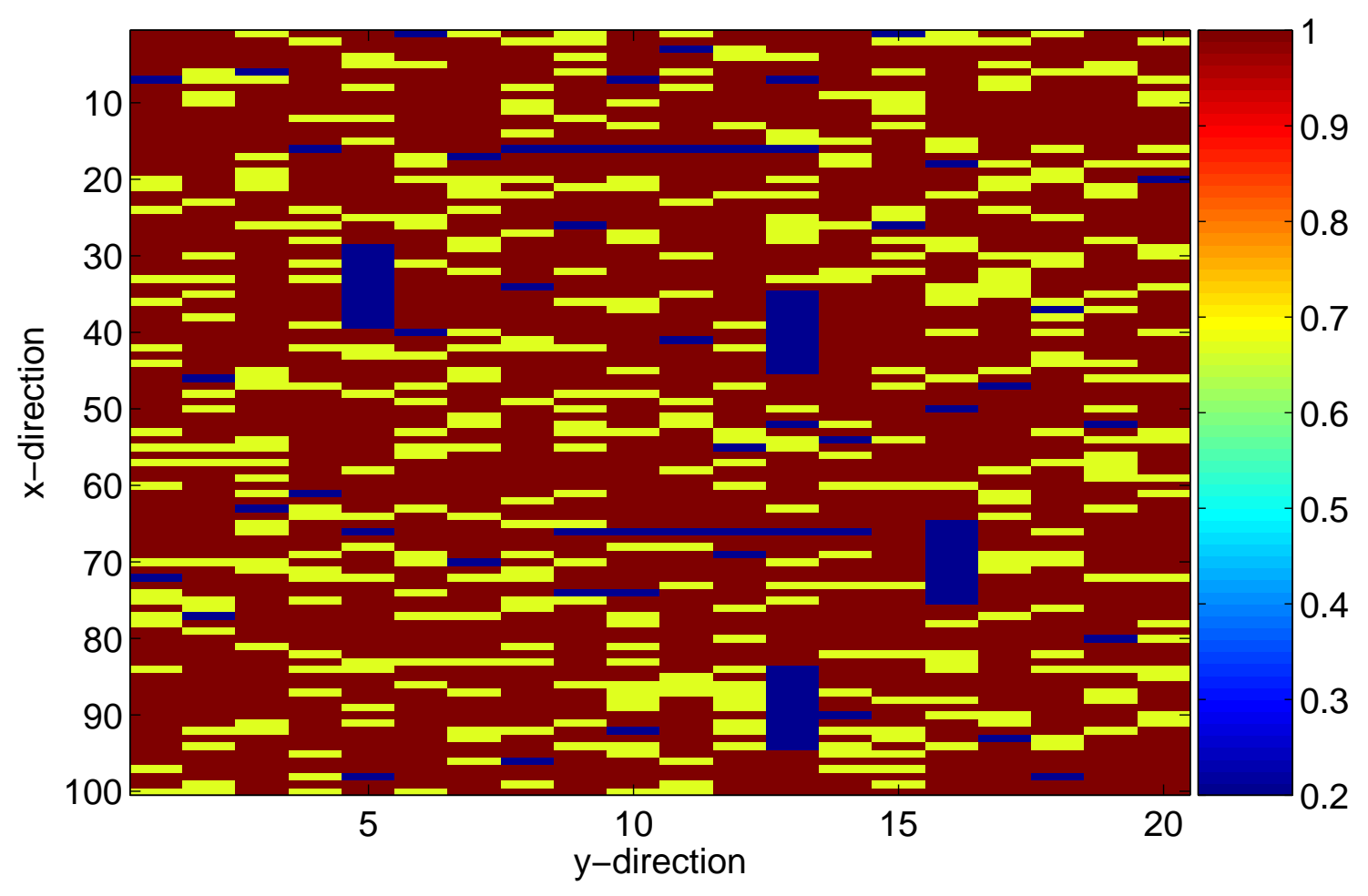

Figure 2. 


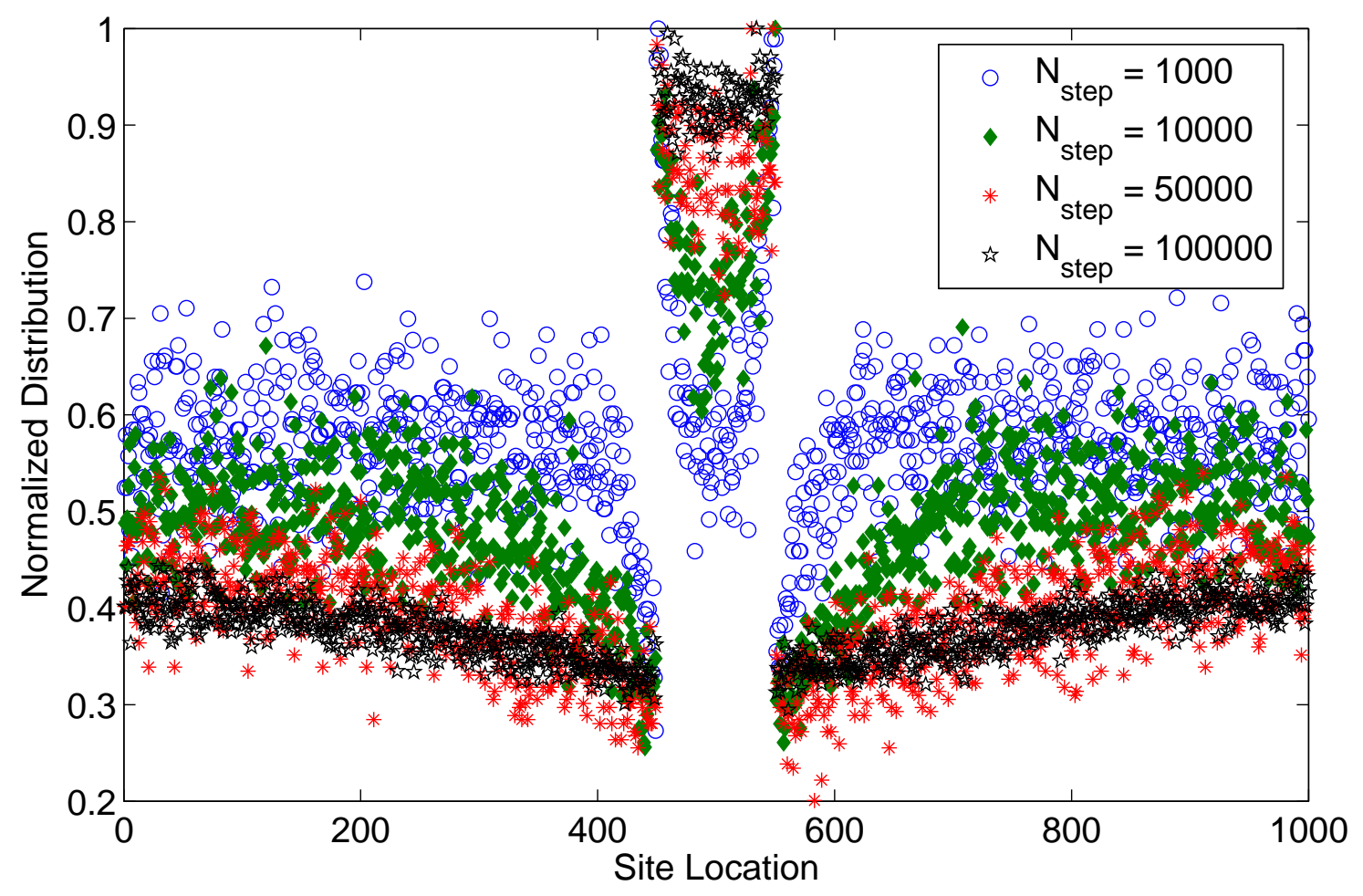

Figure 3.

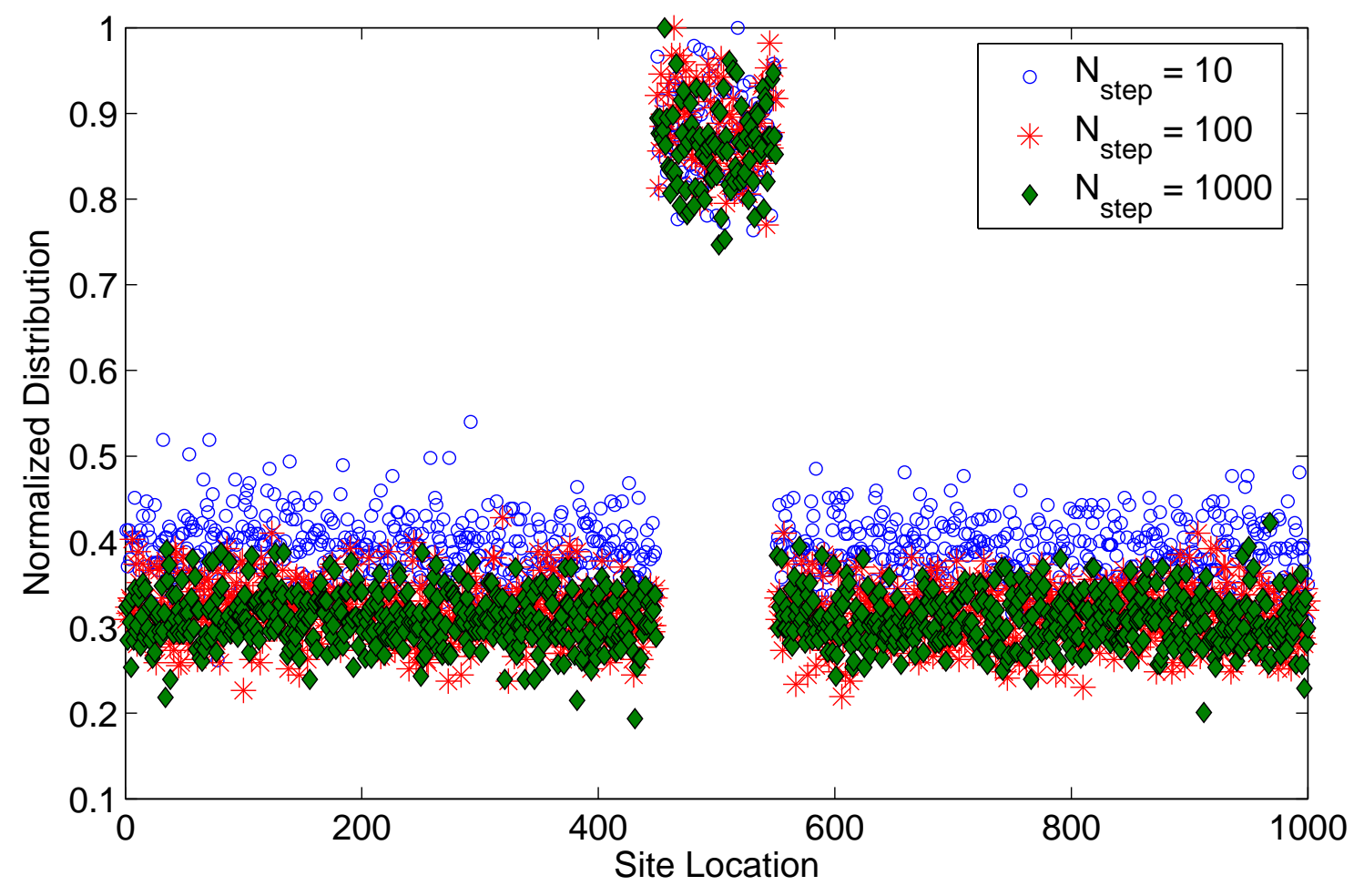

Figure 4. 
(a)

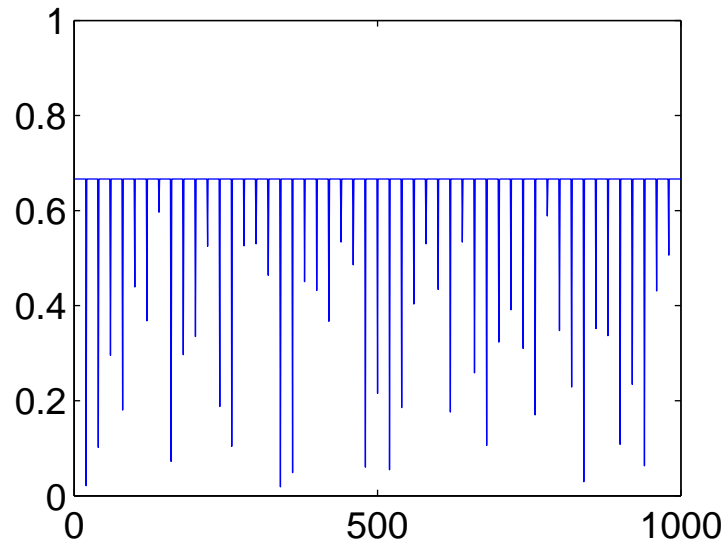

(c)

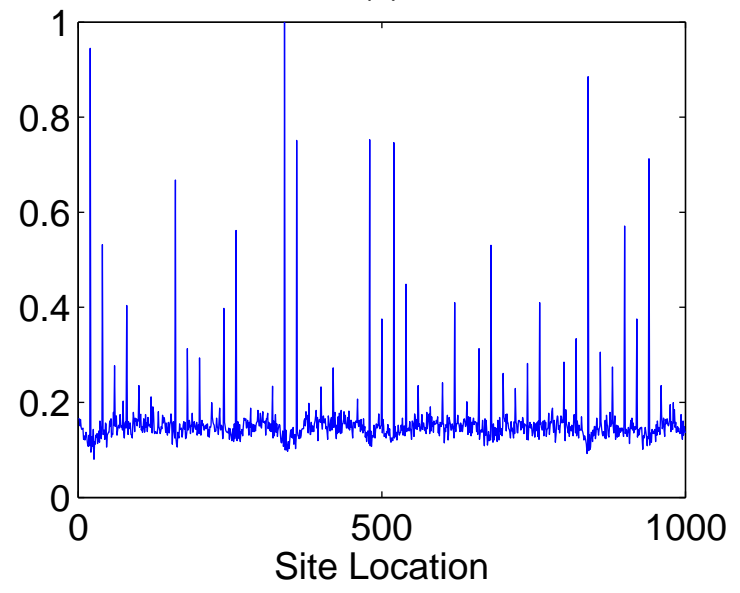

(b)

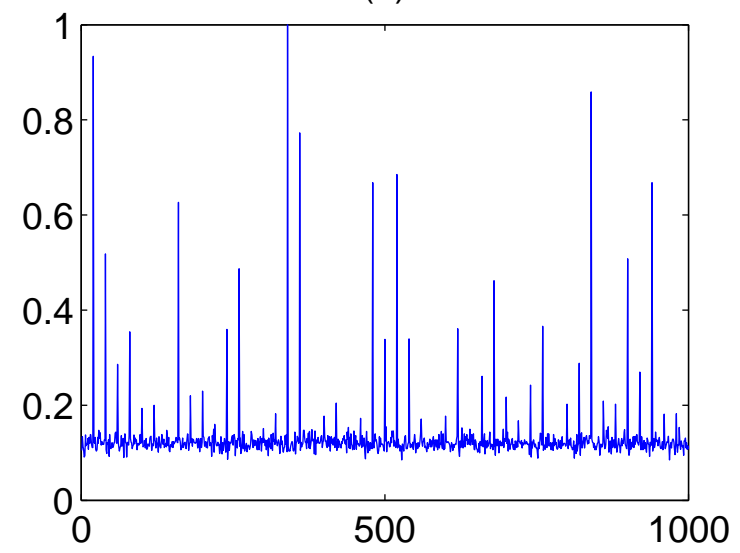

(d)

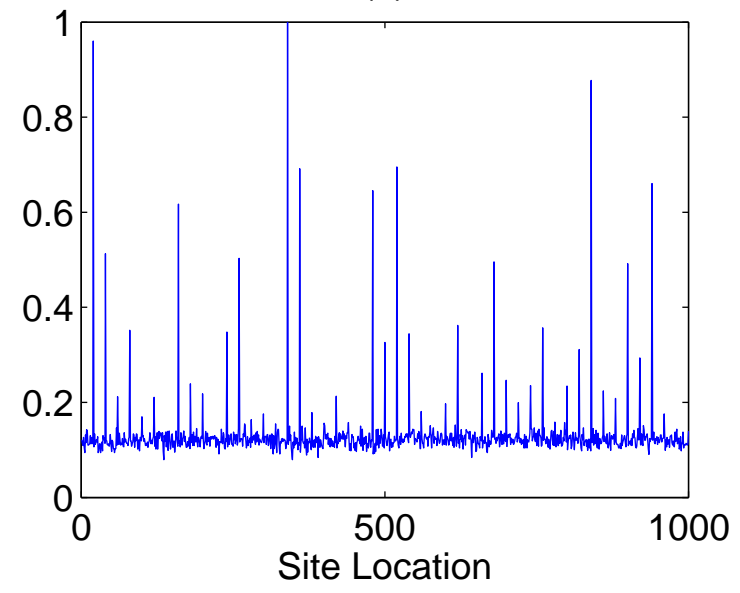

Figure 5. 
(a)

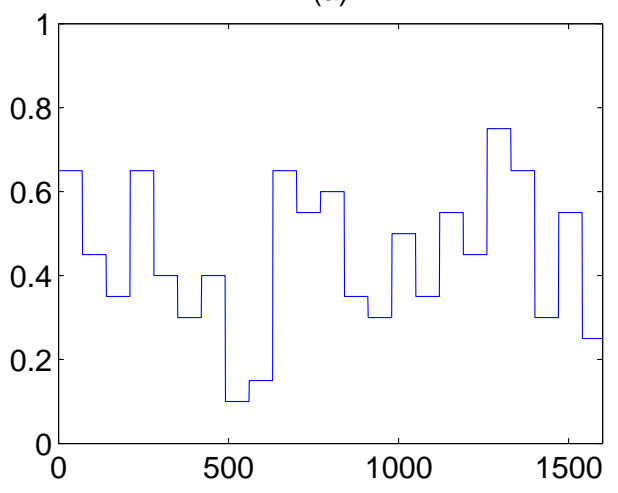

(c)

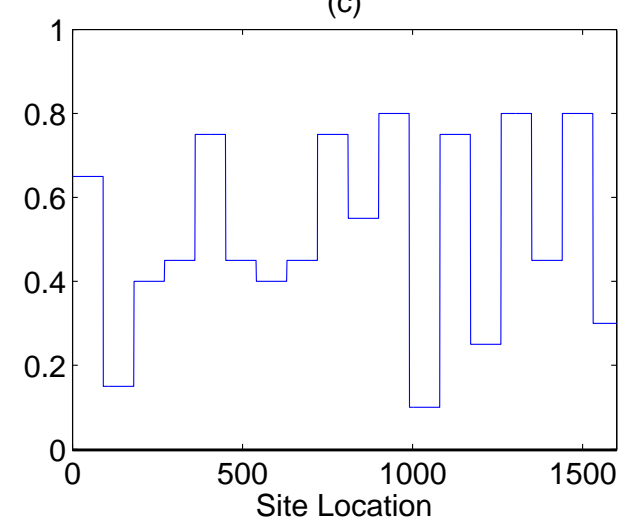

(b)

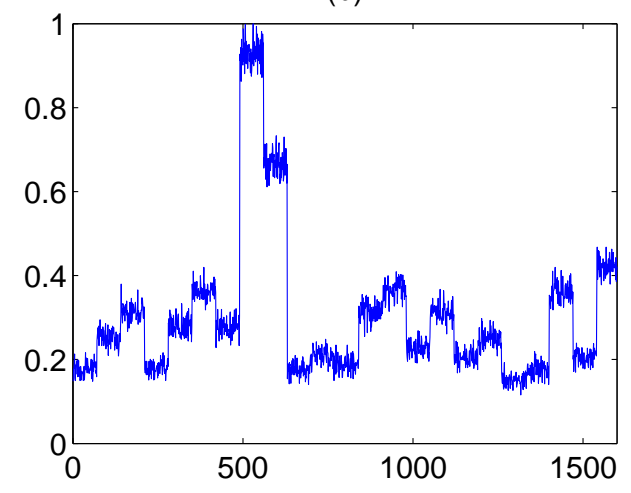

(d)

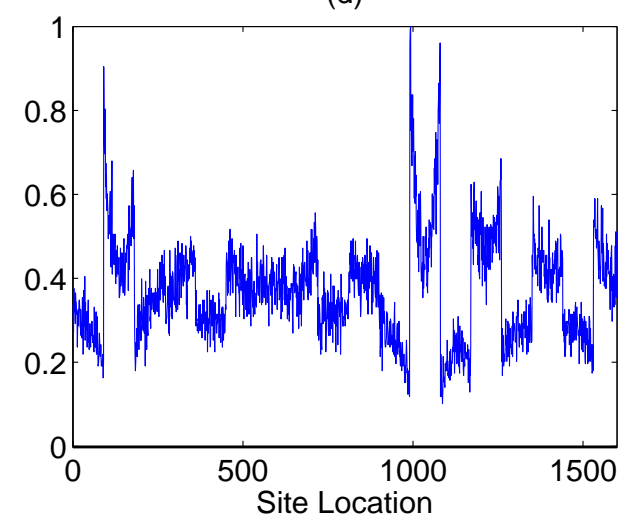

Figure 6. 


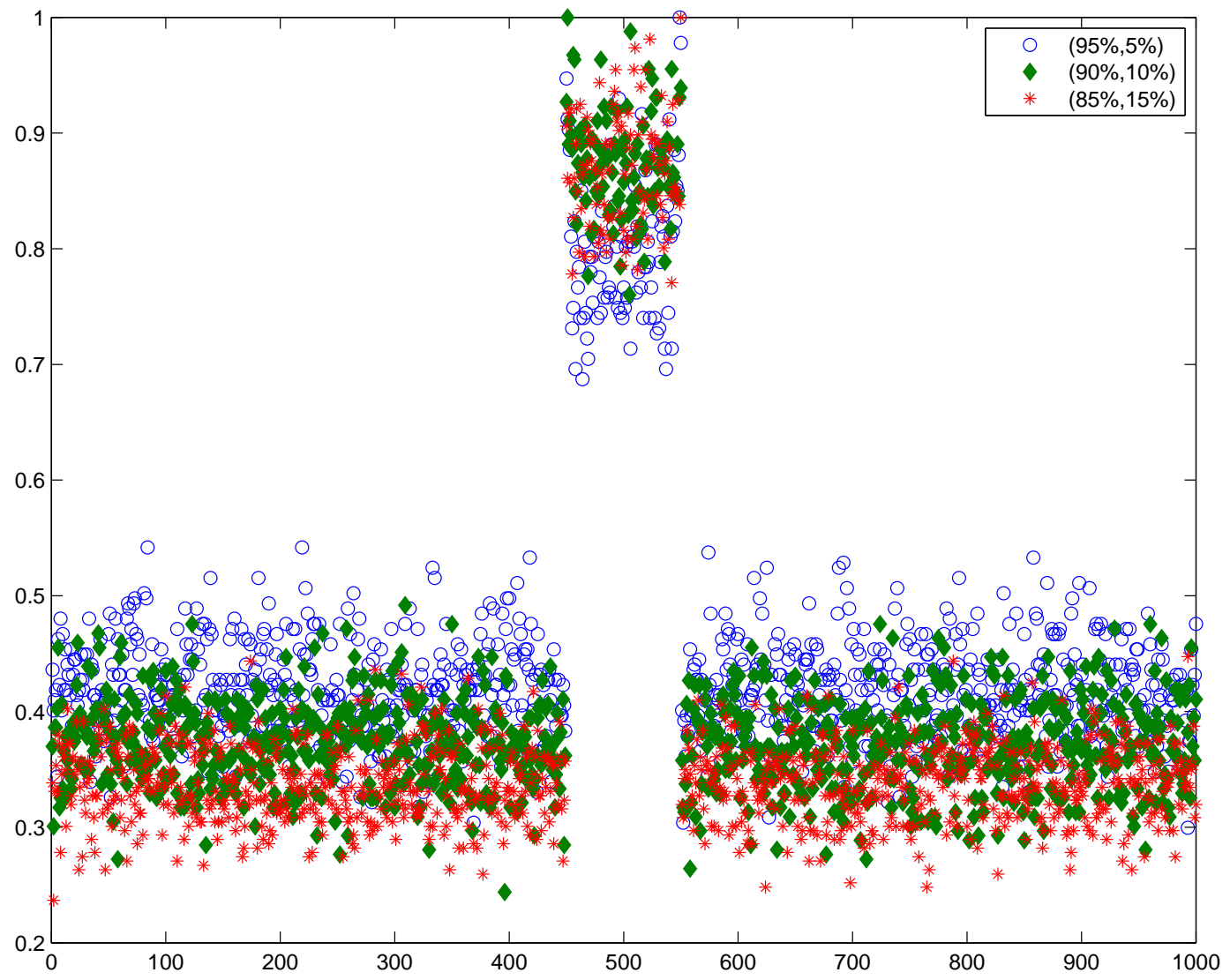

Figure 7. 


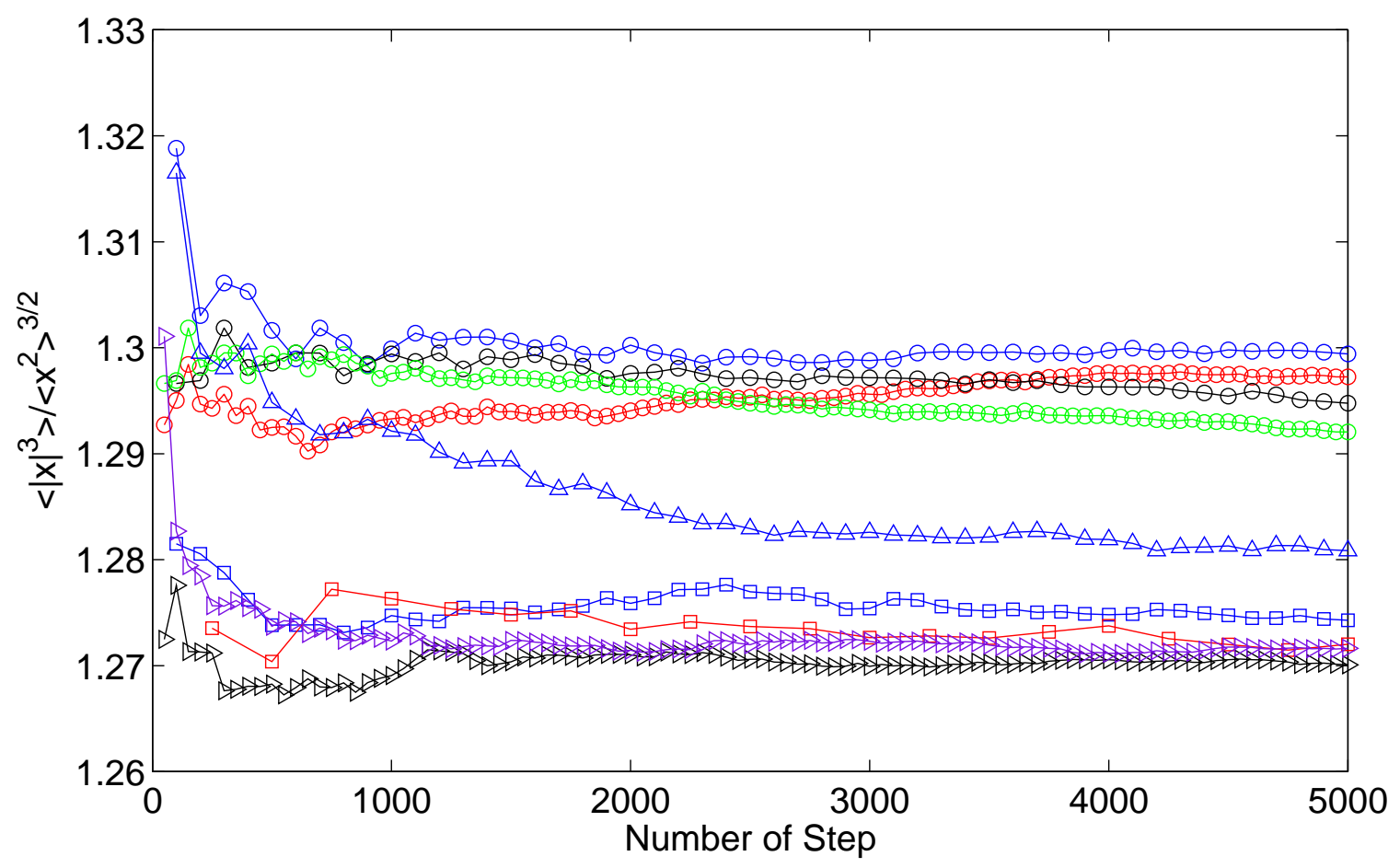

Figure 8.

(a)

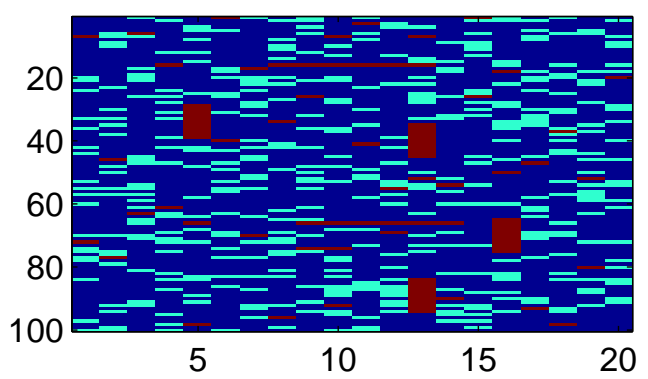

(c)

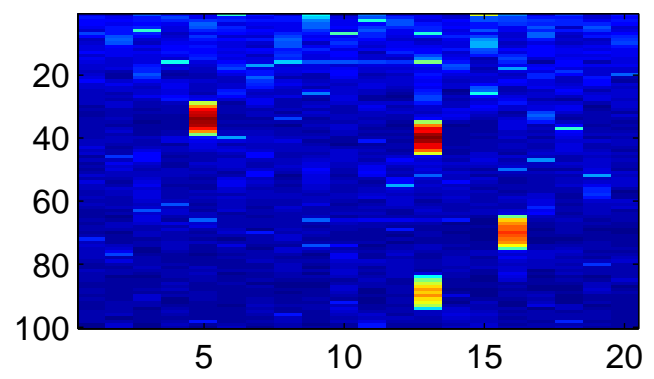

(b)

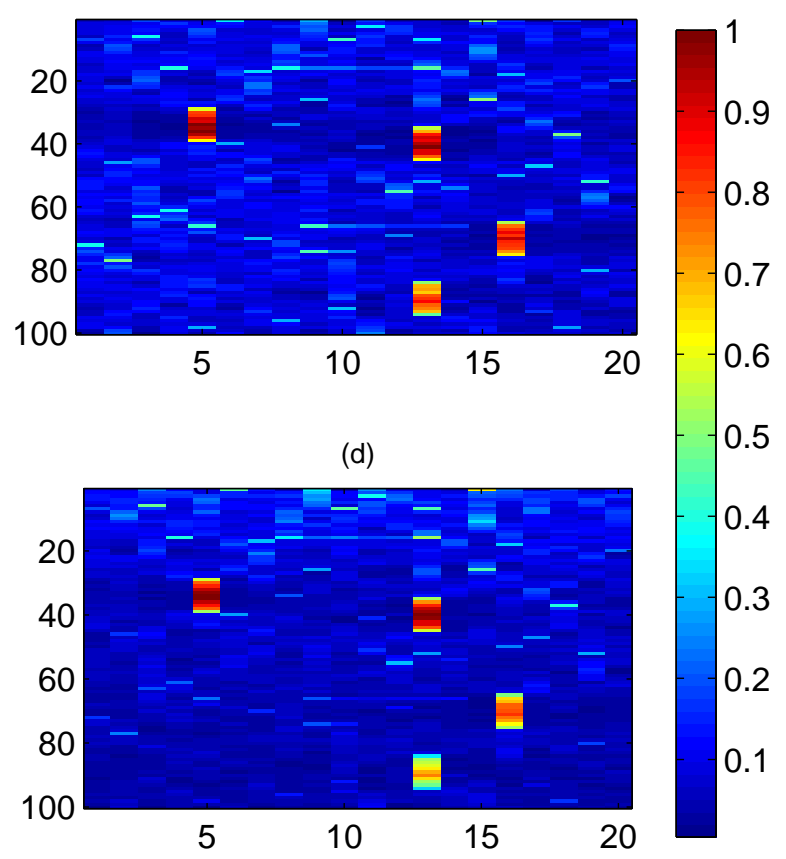

Figure 9. 
(a)

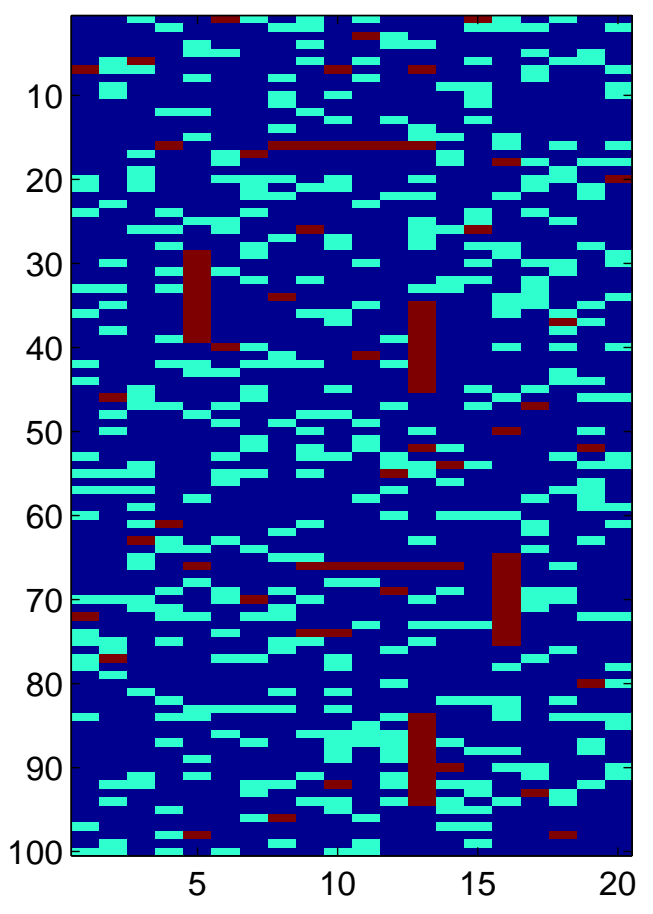

(b)

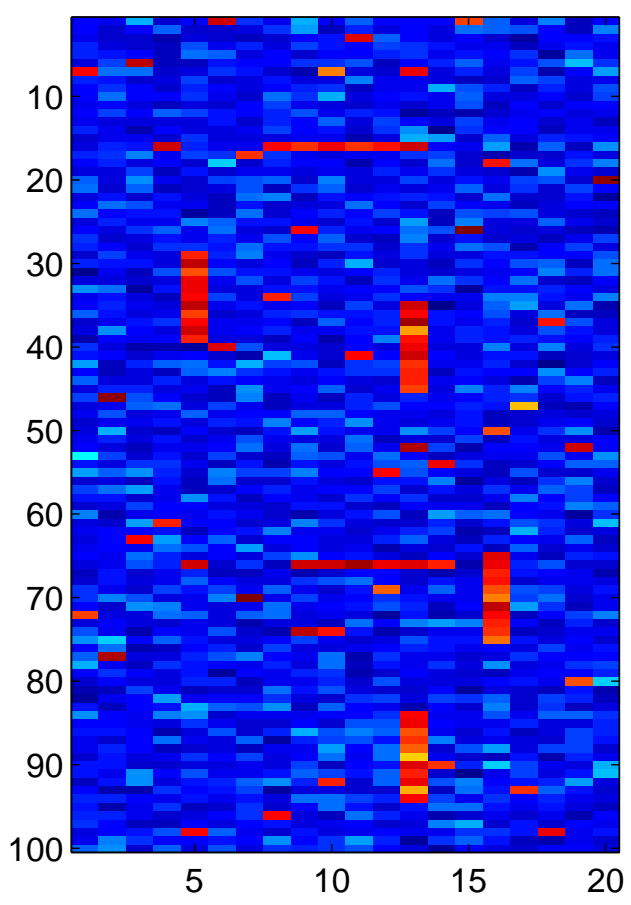

Figure 10. 
(a)

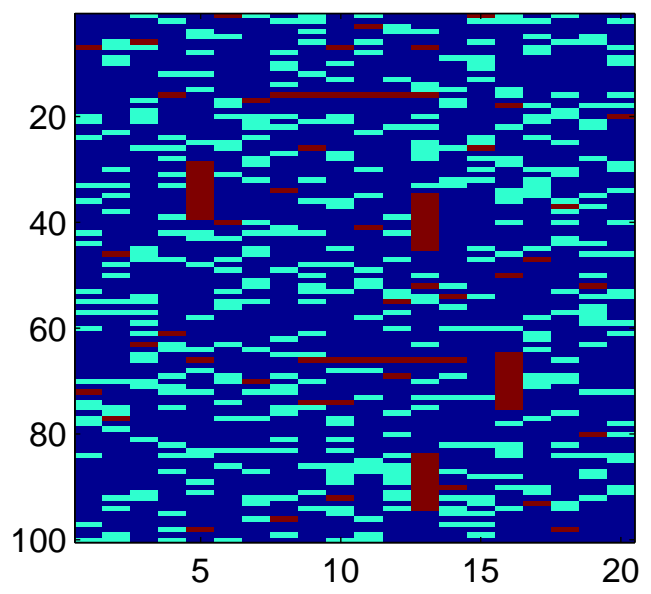

(c)

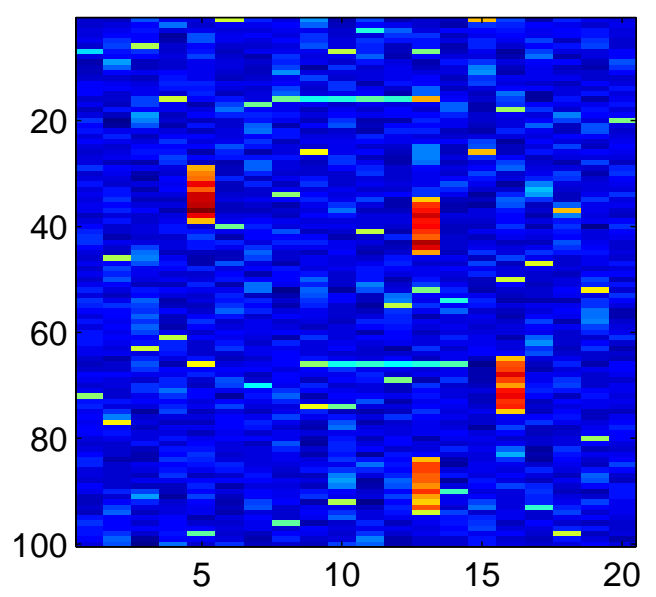

(b)

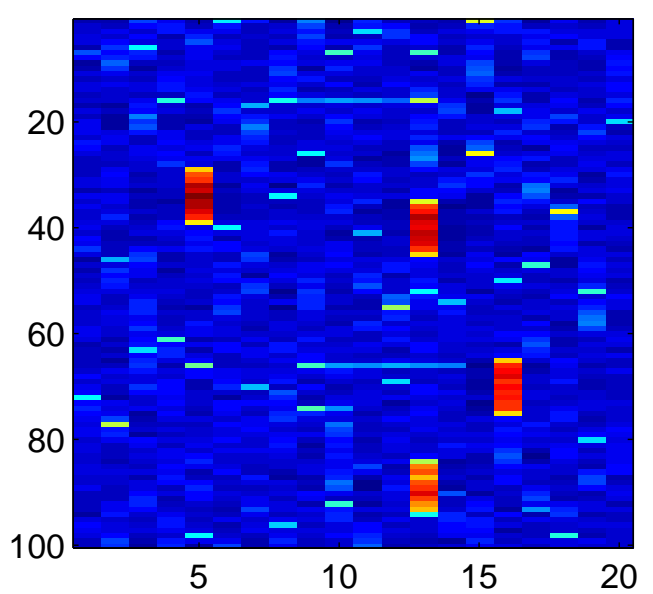

(d)

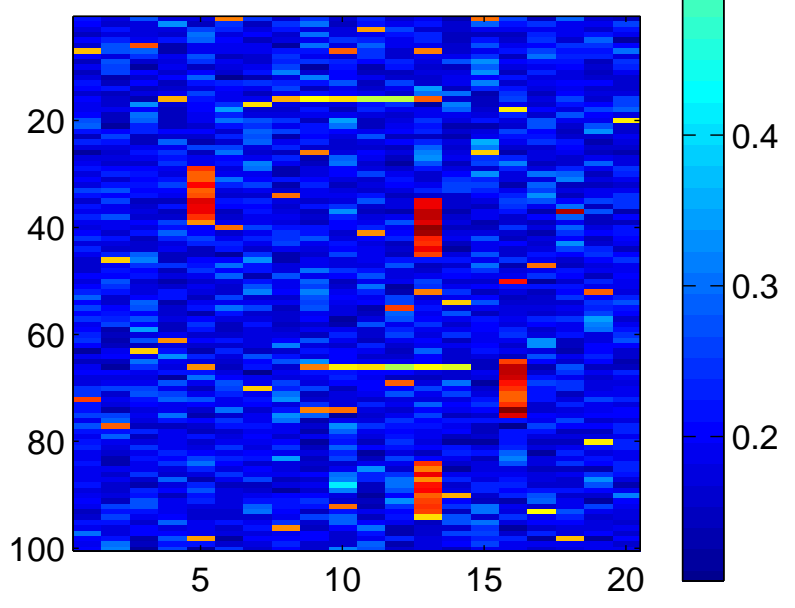

Figure 11. 